\title{
PIXSCAN: Pixel Detector CT-Scanner for Small Animal Imaging
}

P. Delpierre ${ }^{\mathrm{a}}$, F. Debarbieux ${ }^{\mathrm{d}}$, S. Basolo ${ }^{\mathrm{a}}$, J.F. Berar ${ }^{\mathrm{b}}$, A. Bonissent ${ }^{\mathrm{a}}$, N. Boudet ${ }^{\mathrm{b}}$, P. Breugnon ${ }^{\mathrm{a}}$, B. Caillot ${ }^{\mathrm{b}}$, F. Cassol Brunner $^{a}$, B. Chantepie ${ }^{a}$, J.C. Clemens ${ }^{\text {a }}$, B. Dinkespiler ${ }^{\text {a }}$, R. Khouri ${ }^{\mathrm{a}}$, I. Koudobine ${ }^{\mathrm{a}}$, V. Mararazzo ${ }^{\mathrm{d}}$, C. Meessen ${ }^{\mathrm{a}}$, M.

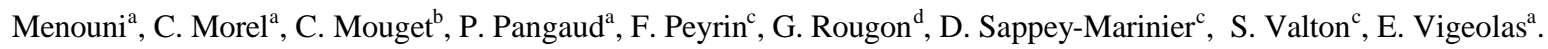

${ }^{a}$ Centre de Physique des Particules de Marseille(CPPM), IN2P3/CNRS, Université de la Méditerranée, France

${ }^{\mathrm{b}}$ CNRS Grenoble \& D2am CRG, France

${ }^{\mathrm{c}}$ CREATIS, UMR-CNRS-5515, INSERM-U630, Lyon, France

${ }^{\mathrm{d}}$ Institut de Biologie du Développement de Marseille Luminy (IBDML), France

\begin{abstract}
The PIXSCAN is a small animal CT-scanner based on hybrid pixel detectors. These detectors provide very large dynamic range of photons counting at very low detector noise. They also provide high counting rates with fast image readout. Detection efficiency can be optimized by selecting the sensor medium according to the working energy range. Indeed, the use of CdTe allows a detection efficiency of $100 \%$ up to $50 \mathrm{keV}$. Altogether these characteristics are expected to improve the contrast of the CT-scanner, especially for soft tissues, and to reduce both the scan duration and the absorbed dose. A proof of principle has been performed by assembling into a PIXSCAN-XPAD2 prototype the photon counting pixel detector initially built for detection of X-ray synchrotron radiations. Despite the relatively large pixel size of this detector $\left(330 \times 330 \mu \mathrm{m}^{2}\right)$, we can present 3D tomographic reconstruction of mice at good contrast and spatial resolution. A new photon counting chip (XPAD3) is designed in sub-micronique technology to achieve $130 \mathrm{x} 130 \mu \mathrm{m}^{2}$ pixels. This improved circuit has been equipped with an energy selection circuit to act as a band-pass emission filter. Furthermore, the PIXSCAN-XPAD3 hybrid pixel detectors will be combined with the Lausanne ClearPET scanner demonstrator. CT image reconstruction in this non-conventional geometry is under study for this purpose.
\end{abstract}

PACs:

Keywords: Hybrid pixels, photon detector, photon counting, CT-scanner

*Corresponding author. Tel.: +33-491827200, Fax: +33-491827299, E-mail: delpierre@cppm.in2p3.fr

Postal address: CPPM, 163 av. de Luminy, BP 902, 13288 Marseille Cedex 9 


\section{Introduction}

Imaging systems dedicated to small animal imaging are now recognized as important tools for both cancer and genomic researches. Actually these new imaging systems allow non-invasive visualization of tissues in rodents, which is of considerable interest to follow evolution of tumors and related therapeutic actions, but also to screen large population of genetically modified animals. One of these modalities, the CT-scanner is a very promising technique since it is a fast and relatively low cost means to characterize tissues organization and skeleton abnormalities. Moreover tumors and vascular system can also be visualized on live animals after injection of contrast agents. The aim of the PIXSCAN project is to develop a CT-scanner offering optimized contrast resolution in soft tissues of mice. In a later stage, plans are to combine this CT scanner with a Positron Emission Tomography (PET) scanner, to allow simultaneous PET/CT imaging of small animals.

The originality of our approach resides in the use of hybrid pixel detectors. These detectors have been developed at CPPM for vertex trackers of high energy physic experiments (DELPHI, ATLAS, CERN, Geneva), and for this reason they are optimized for photons detection and counting. They offer high detection efficiency with low noise and practically infinite dynamic range. In addition, image acquisition and image readout can be done at very high rate (up to $500 \mathrm{~Hz}$ ). These properties are expected not only to improve contrast resolution of images as compared with images obtained with standard CCD based detectors, but also to allow reduction of irradiation dose during a scan.

To evaluate the benefits of hybrid pixel detectors in the context of small animal imaging, we have built a large surface detector with the XPAD2 chips [1] and have assembled a first prototype of CT-scanner (named PIXSCAN-XPAD2). We present here the first projection images and corresponding tomographic reconstructions acquired from living mice with the PIXSCAN-XPAD2 system. These encouraging results led us to build an improved version of detector based on the new XPAD3 chip, with enhanced robustness and reproducibility across repeated imaging sessions. Accurate double energy threshold has been implemented as a new feature to allow narrow energy windowing at the detector level.

Because XPAD3 circuits have been designed to fit with $130 \times 130 \mu \mathrm{m}^{2}$ pixels, the new version of PIXSCAN using XPAD3 will also benefit from improved spatial resolution. We are currently designing equipment to improve mouse conditioning and are studying the optimal configuration to combine the XPAD3 detector with a small animal PET scanner.

\section{The XPAD2 detector.}

The XPAD2 detector is built using the XPAD2 chip [1]. This chip includes 600 pixels of $330 \times 330 \mu \mathrm{m}^{2}$. It includes a charge amplifier, a discriminator and a 15 bits counter. 8 XPAD2 are bump-bonded on a $65 \times 8 \times 0.5 \mathrm{~mm}^{3}$ silicon sensor. Because each chip presents photon insensitive areas on its periphery, these 8 modules have been mounted like tiles, to minimize the dead area. This procedure resulted in a $6,5 \times 6,8 \mathrm{~cm}^{2}$ detector of 36800 pixels, as shown on figure 1 . Thanks to a fast readout system, the full detector can be read in less than $2 \mathrm{~ms}$. This detector has been tested on the D2AM beam of the ESRF (Grenoble) synchrotron in the context of X-ray experiments for material sciences. Both its very large dynamic 
range and fast readout could be demonstrated and appreciated since experiment duration was shortened as much as 10 to 100 times as compared to the standard protocol [2].

\section{Fabrication of the PIXSCAN.}

To evaluate the performances of the XPAD2 hybrid pixel detector for small animal imaging, we have built a very simple CT-scanner prototype. It consists of a step by step rotating animal holder placed between a fixed X-ray source and the fixed X-ray detector. To maximize the body region imaged at once, we have used a wide conic beam aperture.X-ray source $\left(60 \mathrm{kV}, 30 \mathrm{~W}, 0,8 \mathrm{~mA}\right.$; Rontgentek, SEPH, France) with an emission spot of $50 \mathrm{x} 50 \mu \mathrm{m}^{2}$. This configuration illustrated on the figure 2 allows for 3D tomographic imaging of half of the mouse body at once.

The system is installed inside a shielding box made of $5 \mathrm{~mm}$ thick iron plates to protect users from radiations. Most mechanical actions have been remote controlled: in particular mouse is held on a remotely controlled rotating platform with an adjustable angular step (microcontrol, France) and this platform can also be remotely translated to bring the mouse out of the beam for white calibration image acquisition. Finally an external remote shutter has been installed in front of the source to perform the black calibration image.

\section{Mouse conditioning}

Mice were anesthetized using intraperitoneal injection of urethane at a dose of $1.5 \mathrm{~g} / \mathrm{kg}$. They were then placed freely breathing in the imaging system. A $2 \mathrm{~cm}$ diameter plexiglas cylinder was first used to contain mouse body. But although body and legs were nicely maintained this way, images turned out to be blurred due to the surrounding plexiglas shell. The image quality was improved with the animal hung by a mouth piece along a carbon axis, with mouse bump supported on the base (Figure 3). An extra piece of plastic foil was wrapped around the trunk of the animal to avoid movements of the paws during rotation. Advantage of the method resides in the fact that tomographic reconstruction is minimally degraded by mouse support.

\section{Detector tuning and image processing}

Prior to any image acquisition, pixels threshold have to be adjusted one by one. To this end the same electric charge is first injected via the calibration capacitor at the input of the analog front end. Threshold adjustment is then finalized using the X-ray source photoelectric peaks as references. Several black calibration images (no object and source blocked) are acquired and averaged to obtain the map of unstable oscillating pixels. Similarly several white calibration images (no object but source opened) are used to map non-working pixels with constant output. All defective pixels (oscillating and non-counting) are masked for calculation of the tomographic reconstruction.

The white calibration image is also used to normalize each mouse projection image, thereby compensating for residual threshold misalignment after calibration. Normalization is done by dividing each mouse image by the white calibration image. Images are then interpolated to compensate for bad pixels and further filtered using a standard median. Figure 4 gives an example of the images after processing. 


\section{3D Tomographic reconstruction.}

3D tomographic reconstruction of the mouse body at best possible resolution given the detector pixel size requires about 400 cone beam projections. For convenience, we acquired one image per degree (360 images per scan). These images have then been processed using a FDK based reconstruction algorithm for cone beam tomography provided by CREATIS (RecFDK). Examples of mouse tomographic images are presented Figure 5. For these images, the mouse was alive and held hung anesthetized on its carbon tube (seen on the left part of the horizontal slice in Figure5). Although images are still preliminary and can still be improved by corrections taking into account some defects of the detector such as the geometrical tile and gap effects, high absorption contrast can be seen between the air-filled lungs (dark) and the dense spine and rib bones (white) on the horizontal and coronal slices.

\section{Geometrical parameters.}

Geometrical parameters are determined precisely using a $2 \mathrm{~cm}$ diameter cylinder with 3 small metal balls glued on its surface at known positions. Centers of the balls can be easily determined from the projections by fitting an ellipse on high contrast edges. The fitted position of the ellipses are then used to map the trajectories of the centre of the spheres. These trajectories are finally used to determine the exact position of the detectors and of the source with regards to the rotation axis. This is done by minimizing the residuals between the measured position and the estimated positions of the balls for each rotation angle, using the least mean square method. The resulting mean dispersion is about $10 \mu \mathrm{m}$.

\section{Prospects.}

A new version of the PIXSCAN (named PIXSCAN-XPAD3) is under development with the aim to improve the spatial resolution and to make full body scans with a larger detector. To this, we have designed a new chip named XPAD3 [3] with pixels of $130 \times 130 \mu \mathrm{m}^{2}$, precise threshold adjustment, and very fast frame readout $(<2 \mathrm{~ms})$. Moreover, the XPAD3 has been equipped with an energy selection circuit to act as a band-pass emission filter. We expect this feature to be useful in the presence of iodinated contrast agents. Indeed, one would be able to select an energy window lower and higher than the iodine photoelectric absorption jump and, by image subtraction, to enhance the contrast of the regions containing iodine.

The size of the XPAD3 is $10 \times 17 \mathrm{~mm}^{2}$. Modules of 7 chips will be built on silicon and 8 modules will be assembled to form a detector of $12 \times 7,5 \mathrm{~cm}^{2}$. This XPAD3 detector will replace the XPAD2 to build the PIXSCAN-XPAD3. Moreover in this PIXSCAN, the photon detector and the source will rotate around the mouse support.

To improve the detection efficiency we will also build modules with CdTe, but they will be smaller due to the lack of large size CdTe wafers. Modules of 2 chips will be built and $3 \times 4$ modules will be assembled to form a $6 \times 6 \mathrm{~cm}^{2}$ detector.

Beside the development of these XPAD3 detector, tests of simultaneous detection of $\mathrm{X}$ and gamma rays are done with the ClearPET demonstrator [4] and the XPAD2 detector, to study the feasibility of a simultaneous PET/CT for small animal imaging. CT image reconstruction in this non-conventional geometry is under study for this purpose [5]. 


\section{References.}

[1] P. Delpierre et al.," Large surface X-Ray pixel detector (2002). IEEE Trans. on Nucl. Science", 49(4), 1709-1711.

[2] JF. Bérar et al., "A pixel detector with large dynamic range for high photon counting rates". (2002). J. Appl. Cryst. 35, 471-476.

[3] P. Pangaud et al., "XPAD3: A new photon counting chip for X-ray CT-Scanner", presented at this conference.

[4] M. Rey et al., "Measured and simulated specifications of the Lausanne ClearPET scanner demonstrator," in Conf. Rec. of the IEEE Med. Imag. Conf., San Juan, Puerto Rico, Oct. 2005

[5] S. Valton," Evaluation of tomographic reconstruction methods for small animal microCT and microPET/CT ", presented at this conference.

\section{Figure captions:}

Fig. 1. The XPAD2 detector.

Fig. 2. The PIXSCAN ready for the scan of a mouse.

Fig. 3. Mouse holder

Fig. 4. Examples of images (projections) of a mouse.

Fig.5. Tomographic reconstruction of mice images. Left: horizontal slice of a mice thorax, middle: coronal slice, right: bone surface image of a mouse head and thorax 


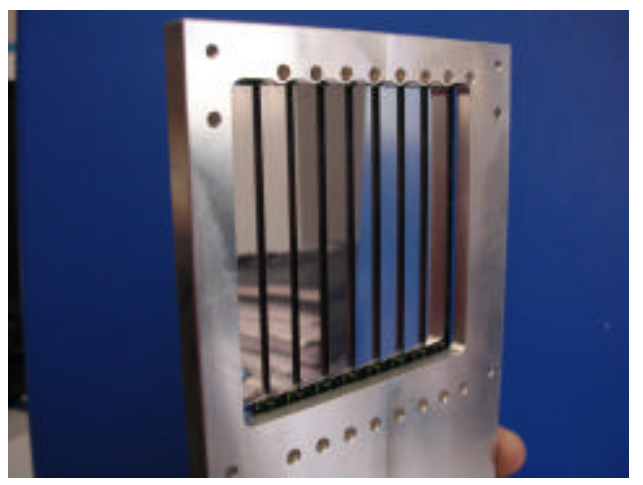

Fig. 1 


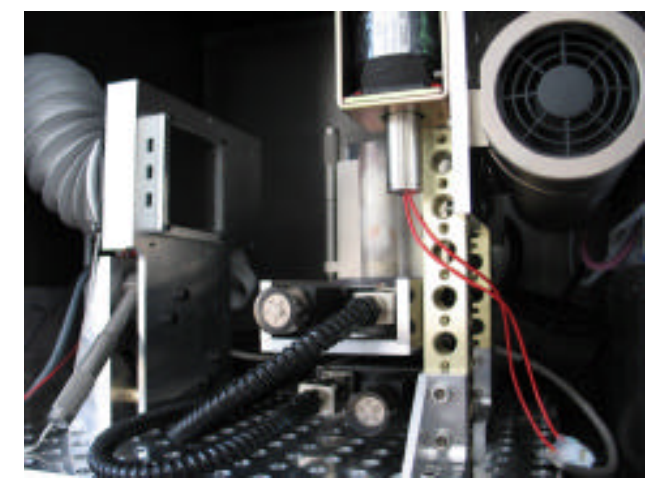

Fig. 2. 


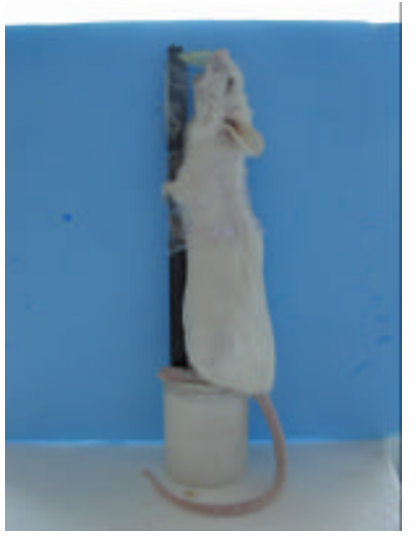

Fig. 3. 


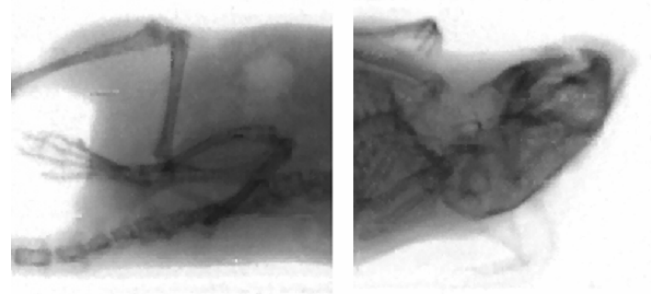

Fig. 4. 


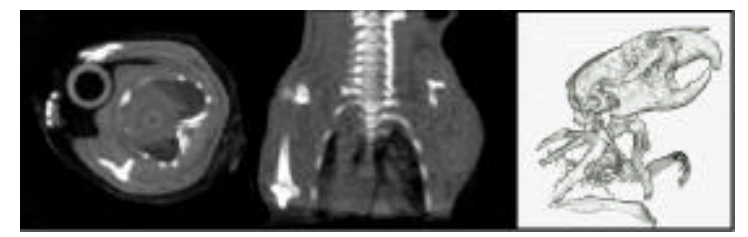

Fig.5 